\title{
Gobierno corporativo: una revisión de literatura especializada
}

\author{
Natalia Jara Pinilla \\ Facultad de Economía y Negocios \\ Universidad de Chile \\ njara@fen.uchile.cl \\ Mauricio Alejandro Jara Bertín \\ Facultad de Economía y Negocios \\ Universidad de Chile \\ mjarab@fen.uchile.cl
}

\begin{abstract}
The governance of the company has been a topic that has gained increasing importance in the corporate world. Although its beginnings in terms of formal discussion date back to the mid-70, the underlying concept has its roots long back in time. The problem originates from the agency theory, which exposes a conflict of interest between shareholders and suppliers.

There are several definitions of Corporate Governance, all pointing to a system of internal and external mechanisms that mitigate the agency problem among the different participants of the large modern company. Among some factors that can shape the significance of relationships within the company are legal systems, which give rise to the relative importance of both external mechanisms, as the market, and internal mechanisms such as the board of directors.
\end{abstract}


Keywords: Corporate Governance; Literature Review; Agency Theory.

\section{Resumen}

El gobierno de la empresa ha sido un tema que ha ganado cada vez más importancia en el mundo corporativo. Pese a que sus comienzos en términos de discusión formal datan de mediados de los años 70 , el concepto subyacente tiene sus raíces de mucho antes. El problema se origina por la teoría de agencia, que expone un conflicto de interés entre accionistas y proveedores.

Existen varias definiciones de gobierno corporativo, pero todas apuntan a un sistema de mecanismos internos y externos que atenúan el problema de agencia entre los distintos participantes de la gran empresa moderna. Entre algunos factores que pueden configurar la seriedad de las relaciones dentro de la empresa se encuentran los sistemas legales, los que darán pie a la importancia relativa que tengan tanto mecanismos externos como el mercado, y mecanismos internos, como lo es el directorio.

Palabaras clave: Gobierno corporativo; revisión de literatura; teoría de agencia

\section{Introdución}

En un comienzo Berle y Means (1932) señalaron que las corporaciones estaban creciendo de tal manera, que la propiedad y el control se estaban separando, idea planteada por Adam Smith en 1776 (Smith, 1776). La justificación a esta afirmación radica en que los accionistas de las empresas transadas públicamente llegan a ser tantos, que no son capaces en conjunto de tomar decisiones diarias para la correcta operación del negocio.

Ahora bien, en momentos donde los intereses entre dueños y controladores no coinciden, hay un potencial conflicto de interés entre ellos. Este conflicto de interés, combinado con la incapacidad 
de la creación de contratos perfectos sin costos o monitorear al agente controlador, reduce el costo de la firma.

La literatura es extensa en abordar los gobiernos corporativos en Estados Unidos, ya que entre 1970 y 1980 los estudios se enfocaron solamente en este país. En este periodo, se caracterizaban por la idea de fuertes administradores y accionistas débiles. Los administradores eran leales a la corporación y no se veían como agentes de los accionistas.

Para los años 90, los administradores empezaron a aceptar el concepto de "valor de los accionistas", lo que se convirtió en una ideología en las corporaciones americanas.

En el nuevo siglo, con escándalos como ENRON y Worldcom, hicieron de los gobiernos corporativos un nuevo foco de estudio. Investigadores han utilizado estos eventos como críticas al sistema basado en la gestión empresarial. Los políticos reaccionaron y desarrollaron la ley Sarbanes-Oxley Act (SOX) y enseguida la SEC cambió los requerimientos de las empresas para transar en bolsa, lo que ha alterado la forma en que operan los gobiernos corporativos.

\section{Gobierno corporativo}

A. ¿Qué es gobierno corporativo?

Existen muchas definiciones de gobierno corporativo, sin embargo, el concepto es relativamente nuevo.

Como punto de partida, tenemos la idea postulada por el Cadbury Report (1992), donde define gobierno corporativo como un sistema por el que las empresas son dirigidas y controladas.

Por otro lado, con una definición levemente diferente, pero que apunta a los mismos principios, encontramos a Hart (1995), que afirma que el concepto de gobierno corporativo nace de la existencia de un problema de agencia y la existencia de costos de transacción 
asociados a este, que intervienen en la creación de contratos bien definidos, haciendo de estos una tarea muy difícil.

Una mirada más funcional de lo que es gobierno corporativo la da Merton y Bodie (1995). Este enfoque reconoce que los servicios financieros tienen muchas formas, pero que si están desagregados, pueden tener altas similitudes. Además, este enfoque distingue seis funciones de los gobiernos corporativos: poner en marcha los recursos y dividir las acciones, transferir recursos a través del tiempo y el espacio, manejar riesgos, generar y proveer información, lidiar con problemas de incentivos y resolver competencias generadas por las riquezas corporativas.

Shleifer y Vishny (1997) definen gobierno corporativo como un conjunto de mecanismos encargados de asegurar a los proveedores de recursos de la firma, la obtención de los retornos de sus inversiones. Estos mecanismos son tanto legales como económicos.

Una idea más amplia la brinda Rajan (1998), que entiende este concepto, en el escenario de contratos incompletos, como un conjunto de mecanismos y restricciones que intentan regular las negociaciones ex post de las cuasi rentas generadas por las empresas.

Otra definición que encontramos en la literatura la ofrece Denis y McConnell (2003), que definen gobierno corporativo como el conjunto de mecanismos, institucionales y de mercado, que inducen al interés propio de los controladores de una compañía, a tomar decisiones que maximizan el valor de esta y por consiguiente maximizan el valor para los accionistas.

La OCDE (2004) define gobierno corporativo como los medios internos por las que las corporaciones son operadas y controladas.

Claessens y Yurtoglu (2012) afirman la existencia de dos categorías de definición. La primera definición se enfoca en los patrones de comportamiento, es decir, el verdadero comportamiento de las corporaciones medido en desempeño, eficiencia, crecimiento, estructura financiera y trato a los accionistas y partes interesadas. 
La segunda categoría se enfoca en la estructura normativa de la corporación, las reglas bajo las cuales las firmas operan y las reglas provenientes de otras fuentes, como el sistema legal, el mercado financiero y el mercado laboral. Esta definición es más relevante para estudios comparativos.

Entonces la pregunta inherente a la segunda definición es ¿cuán amplia se debe definir la estructura de gobiernos corporativos?

Los autores responden esta pregunta bajo una definición bien específica de la provisión de financiamiento, donde el foco debe ser en cómo los inversionistas externos se protegen de los inversionistas internos. Esto puede incluir la protección a los derechos de los minoritarios y acreedores.

Independiente de la definición que escojamos para gobierno corporativo, podemos concluir que todas ellas tienen similitudes, basadas en mecanismos, internos o externos, que guían el control de las organizaciones a la maximización de los recursos y aumentar el valor de la empresa, alineando los intereses de los directivos y accionistas.

\section{B. Historia del gobierno corporativo en EE.UU.}

En los años siguientes a la Segunda Guerra Mundial, Estados Unidos experimentó un sostenido crecimiento económico. Las compañías exitosas de la época crecieron muy rápido $\mathrm{y}$, en consecuencia, los accionistas ganaron mucho.

La mayoría de las compañías eran dirigidas por administradores competentes y no por sus dueños, y en este escenario económico muchos ejecutivos abusaron de su poder y solo eran responsables por sus activos. Así, en medio de la prosperidad empresarial, el gobierno corporativo no era prioridad.

A mediados de 1970, luego del colapso de Central Penn, un conglomerado de trenes, los ejecutivos y directores empezaron a 
luchar para mantener el control de los imperios corporativos creados entre 1950 y 1960 . Solo en ese momento se empieza a acuñar el término de gobierno corporativo, como afirma Veasey (1993).

Durante la década de 1980, en Estados Unidos se vivió una gran ola de absorciones y reestructuraciones en el mundo de los negocios, la explicación para este fenómeno es respuesta a las deficiencias de los gobiernos corporativos.

Jensen (1993) argumenta que los cambios en tecnología, en regulación, en competencia y en los mercados de capital, llevaron a la existencia de una excesiva capacidad de las corporaciones en Estados Unidos. Los sistemas internos de control fallaron en el manejo, y el mercado de capital proporcionó las herramientas para eliminar estos excesos. Algunos mecanismos fueron las adquisiciones apalancadas, las compras apalancadas, las compras hostiles y la recompra de acciones.

Donaldson (1994) articula que con el aumento de accionistas institucionales y la mayor cantidad de información disponible en el mercado de capitales, los gobiernos corporativos sienten más presión en aumentar el valor para los accionistas. Sin embargo, en esta década los administradores son leales a la corporación y no a los propietarios, por lo que es denominada "década de confrontaciones".

Según Morck et al. (1989) y Berger y Ofek (1996), las adquisiciones se dan en empresas con bajo valor relativo.

La ola de fusiones y adquisiciones es explicada por un ascenso de los mercados de capital por encima de los gobiernos corporativos. En algunos casos, el mercado de capital ayuda a eliminar exceso de capital y, en otros, disciplina a administradores que no han velado por la maximización de valor para los inversionistas, sino que para otros stakeholders, concluye Kaplan y Zingales (1997).

A principios de la década de 2000, una solución a los problemas de agencia propuesta estaba orientada a la compensación administrativa, pagar altos incentivos a los ejecutivos por un mejor 
desempeño. Sin embargo, los CEO recibían grandes cantidades de dinero, que no tenían precedentes. A esto se sumó los escándalos de ENRON y WorlCom, donde Coffee (2006) argumenta que ambos escándalos tuvieron una característica común. Los altos directivos, que querían obtener beneficios personales mediante las opciones de acciones y las compensaciones por incentivo, manipularon las cifras contables para hacer parecer que sus empresas estaban alcanzando los resultados esperados.

Finalmente, podemos nombrar la crisis Subprime, donde volvemos a encontrar ejecutivos ambiciosos de las principales empresas financieras que tomaron ventajas de juntas directivas y accionistas pasivos (Kirkpatrick, 2009).

\section{Mecanismos del gobierno corporativo}

Denis y McConnell (2003) hacen una categorización de los mecanismos internos y externos de los gobiernos corporativos. Solo se expondrán algunos de ellos.

\section{a. MECANISMOS INTERNOS}

Directorio: En Estados Unidos, su principal función es representar los intereses de los accionistas. En teoría, el directorio es un buen mecanismo interno de gobierno corporativo, sin embargo, en la práctica su valor no es tan claro. En Estados Unidos, los miembros son los mismos a quienes se debe monitorear y en muchos casos el CEO es el que preside la junta. Además, la naturaleza del proceso de selección de los miembros es tal, que los mismos directivos pueden elegir a sus compañeros.

Estructura de propiedad: La propiedad y el control pocas veces están separados en una firma. Los controladores frecuentemente tienen un 
porcentaje del patrimonio de la empresa que controlan. A medida que los intereses de los administradores y accionistas difieren, una mayor parte del patrimonio para los administradores puede otorgarle mayor libertad para perseguir sus propios intereses.

\section{b. MECANISMOS EXTERNOS}

Mercado de adquisición: Cuando los mecanismos internos de la firma fallan, o sea, cuando la brecha entre el valor actual de la firma y su valor potencial es suficientemente negativa, existen incentivos para partes externas a la empresa a buscar su control. La importancia de este mercado es que con solo existir una amenaza de un cambio de control es suficiente para incentivar a los administradores a mantener un alto valor de la empresa.

Sistema legal y su regulación: Jensen (1993) reconoce al sistema legal como un instrumento de mecanismo externo de gobierno corporativo, pero lo encuentra un instrumento demasiado tosco para abordar con eficacia los problemas de agencia entre accionistas y administradores. La Porta et al. (1998) enfatizan que el sistema legal es un importante mecanismo, ya que en la medida que las leyes de un país protejan los derechos de los inversionistas y el grado en que se aplican, son los pilares fundamentales de la forma de las finanzas corporativas y gobiernos corporativos de ese país.

Basado en estos mecanismos, existe una división de los estudios de gobierno corporativo en dos generaciones. La primera generación estudia varios de ellos, pero estos estudios son realizados en países fuera de Estados Unidos.

La primera generación responde dos preguntas principales, la primera ¿los mecanismos afectan el desempeño de la empresa?, donde desempeño se define como medida de rentabilidad o valor 
relativo de mercado, y la segunda pregunta, ¿los mecanismos afectan las decisiones tomadas por la empresa?, por ejemplo la rotación y reemplazo administrativo, políticas de inversión y reacciones a ofertas.

Esta generación revela diferencias significativas en la forma de los gobiernos corporativos en los países estudiados, sin embargo, no explica por qué observamos estas diferencias.

Así nace la segunda generación. La evidencia de la primera generación indica que los accionistas en bloque ${ }^{1}$ son más comunes en países fuera de Estados Unidos y que tienen un efecto más significativo en el desempeño, sin embargo, estos estudios no revelan las razones por el aumento en el impacto de grandes accionistas fuera de Estados Unidos.

Zingales (1994) ayuda a entender esta situación exponiendo el caso de Italia, donde el premio por voto es mucho mayor que en el resto de los países, debido a vacíos legales que no protegen los intereses de los accionistas minoritarios.

Denis y McConnell (2003) concluyen que uno de los aportes más importantes de la segunda generación es que los sistemas legales de los países, en particular los que protegen los derechos de los inversionistas, tienen un efecto fundamental en la estructura de los mercados, en la estructura de gobierno que adoptan las compañías $\mathrm{y}$, finalmente, en la efectividad de esos sistemas de gobiernos corporativos.

La investigación de la segunda generación efectivamente comienza con Law and Finance, de La Porta et al. (1998). Ellos postulan que el grado en que las leyes de un país protegen los derechos de los inversionistas y el grado de ejecución de esas leyes, son determinantes fundamentales en la forma en que corporaciones financieras y gobiernos corporativos evolucionan en ese país.

${ }^{1}$ Accionista en bloque: dueño de una gran cantidad de acciones o bonos de una compañía. Tiene gran poder de votación. 
Por otra parte, la propiedad concentrada puede ser respuesta a la falta de protección de los inversionistas. La Porta et al. (1998) señalan que en situaciones donde la ley no protege a los dueños de los controladores, entonces los dueños buscarán ser controladores. El problema de agencia entre administrativos y dueños, recordando que es el punto de inicio de los gobiernos corporativos en Estados Unidos, no será importante, ya que los grandes inversionistas tendrán los incentivos y la habilidad para controlar a los administradores. Sin embargo, la concentración lleva a un conflicto de agencia entre accionistas mayoritarios y minoritarios.

\section{Entorno legal y gobierno corporativo}

\section{a. PROTECCIÓN LEGAL}

Recientes investigaciones de gobierno corporativo alrededor del mundo han establecido un número de regularidades empíricas. Los diversos elementos financieros de los países como son la amplitud y profundidad de los mercados, el ritmo de los nuevos problemas de seguridad, las estructuras de propiedad corporativa, políticas de dividendos, y la eficiencia de asignación de la inversión, parecen explicarse, conceptual y empíricamente, por lo bien que las leyes en estos países protegen a los inversionistas.

De acuerdo con La Porta et al. (2000), la protección a los accionistas y acreedores por el sistema legal es central para entender los patrones de finanzas corporativas en diferentes países.

La expropiación puede tomar varias formas. En algunos casos, los accionistas internos simplemente roban de las ganancias. En otros casos, los activos u otros seguros, son vendidos por los insiders a empresas que ellos también controlan, bajo el valor de mercado. En casos más extremos, la expropiación se hace mediante el nombramiento de familiares de los accionistas en puestos de alta gerencia. 
La protección de los inversionistas externos termina siendo muy importante, porque en muchos países la expropiación a accionistas minoritarios y acreedores es extensa. Entonces, el alcance legal de los gobiernos corporativos promueve que el mecanismo clave para proteger a los inversionistas externos es el sistema legal, es decir, tanto sus leyes como su aplicación.

Holderness y Sheehan (1991) describen los mecanismos de organización como limitantes de abusos a los accionistas minoritarios por parte de los propietarios mayores, concentrando el derecho a voto en algunos y aumentado su "voz" en la junta directiva.

Además, el tema de la protección a la expropiación tiene grandes implicancias en la estructura de propiedad de las firmas y el control. Cuando los derechos de los inversionistas están poco protegidos y la expropiación es factible, el control es muy importante, ya que da la oportunidad a los insiders de realizar expropiación de manera muy eficiente, así el llamado beneficio privado del control se vuelve una parte sustancial de la empresa.

¿Entonces, el control quedará en manos de unos pocos o estará dividido entre varios inversionistas? Se cree que los empresarios, por muchas razones, se quedarán con el control cuando la protección es pobre. La Porta et al. (1999), afirman que si la expropiación se hace en secreto, el control compartido puede restringir los deseos de los empresarios. En el modelo de Bebchuk (1999), las estructuras de control difusas son inestables cuando los inversionistas pueden concentrar el control sin pagar por ello. Estas son algunas de las razones por las que, los países con protección baja a los inversionistas, necesitan mantener un control concentrado en algunos.

Bennedsen y Wolfenzon (2000) hacen un argumento compensatorio. Cuando la protección de los inversores es pobre, disipando el control entre varios inversionistas grandes, donde ninguno de ellos puede controlar las decisiones de la empresa sin estar de acuerdo con los demás, puede servir como un compromiso 
de limitar la expropiación. Cuando no hay un solo accionista controlador y el acuerdo de varios accionistas es necesario para realizar decisiones importantes, entonces estos inversionistas pueden mantener suficientes derechos de flujos de efectivo para limitar la expropiación a los demás inversionistas y entregar las ganancias en forma de dividendos.

En cuanto a los mercados financieros, la teoría es que la protección a los inversores proporciona el desarrollo de estos mercados. Cuando los inversionistas están seguros pagan más por los valores, lo que motiva a las empresas a emitir más.

El derecho de los acreedores estimula el desarrollo de los préstamos y esta estructura, paralelamente, favorece los préstamos bancarios. Y en cuanto al derecho de los accionistas, estimula el desarrollo de los mercados accionarios, como medida de valoración de las firmas, número de firmas cotizando en bolsa y la velocidad a la que las empresas se hacen públicas.

Becht (1999) examina el intercambio entre liquidez de mercado y control, cuando los mercados no líquidos fuerzan a grandes inversionistas a mantener un monitoreo activo. La ley de protección a los accionistas minoritarios tiene como fin animar la inversión de los accionistas pequeños, haciendo los mercados más líquidos $\mathrm{y}$, por esta razón, disminuyendo el control. El autor argumenta que en la práctica se da un escenario completamente contrario, y que la ley causa efectos competitivos entre las variables estudiadas.

Esta afirmación es consistente con la evidencia encontrada por La Porta et al. (1998) en un estudio a 49 países que concluyó que la calidad de la legislación de protección a los inversionistas y la concentración de propiedad estaban negativamente correlacionadas, ya que la leyes de protección hacen los mercados más líquidos. 


\section{b. COMPARACIÓN LEY COMÚN Y LEY CIVIL}

Los sistemas financieros se pueden clasificar en función del origen del país y, al mismo tiempo, de la cobertura legal de los inversores. Por una parte existe un sistema de raigambre anglosajona o de ley común y, por otro lado, un sistema de ley civil, derivado de las leyes romanas. En cuanto a la tradición civil, hay tres leyes modernas que derivan de ellas: francesa, germana y escandinava.

La ley civil brinda una menor protección a los inversionistas que la ley común, independiente del nivel per cápita que tenga el país. Los países con ley anglosajona son los países con mayor protección (relativa) para los accionistas y acreedores, y los de ley civil francesa, los más débiles.

En vista de estos resultados, que la ley y su aplicación varía de país en país según su familia de procedencia, La Porta et al. (1998) estudiaron cómo países con leyes o aplicación pobre de estas, hacen frente a este problema y si estos países tienen mecanismos sustitutos de gobierno corporativo.

Dentro de este estudio se encontró que la ley común ofrece la mejor protección legal a los accionistas. Ella permite, frecuentemente, ejercer el derecho a votación por correo, tienen la mayor incidencia de las leyes que protegen a los minoristas oprimidos y, en general, se requiere poco capital para llamar a una junta extraordinaria de accionistas. La única dimensión en que la ley común no es especialmente protectora es en el derecho de preferencia para las nuevas emisiones de acciones. La Porta et al. (1998) concluyen que muchas de las diferencias entre la ley común o anglosajona y la ley civil son estadísticamente significativas.

En otro tema de protección legal, la ley común ofrece mejor protección legal para los acreedores por sobre los administradores. Por ejemplo, tiene la mayor incidencia de no suspensión automática de los activos, se opone a los gerentes de buscar unilateralmente la protección judicial de los acreedores. Estados Unidos es, actualmente, el más antiacreedor de los países de ley común: 
permite la suspensión automática de los activos, permite sin obstáculos la petición de reorganización y permite a los administrativos a mantener sus trabajos en las reorganizaciones.

En algunas medidas, los países de ley civil germana son muy proacreedores. Por ejemplo no permiten la suspensión de activos y a los acreedores asegurados se les paga primero.

En uno de los recientes trabajos empíricos de Chung et al. (2012), analizan 25 países, de estos 8 tienen ley común, 8 tienen ley civil francesa, 5 tienen ley civil germana y 4 tienen ley civil escandinava.

Utilizando herramientas estadísticas encontraron diferencias significativas entre los tipos de ley analizados. Países en ley común tienden a tener una mejor liquidez en sus mercados accionarios y mejores puntajes de gobernabilidad que los países en ley civil.

Además, demuestran que las firmas pertenecientes a países de ley común tienen mayor volatilidad de la rentabilidad, mayores volúmenes de comercio y activos, pero una proporción menor de propiedades, plantas y equipos del total de activos, que empresas pertenecientes a países de ley civil.

Estos atributos permiten concluir que las firmas con un sistema legal anglosajón tienen mejores estructuras de gobiernos corporativos y una mayor liquidez de mercados de valores.

\section{c. MERCADOS Y GOBIERNOS CORPORATIVOS}

El primer aspecto interno de la empresa de los gobiernos corporativos a analizar son los derechos de los accionistas. Como la mayoría de los accionistas ejercen su poder votando, la mayoría de los expertos se enfoca en la votación para evaluar los derechos.

Grossman y Hart (1988) afirman que el derecho a votación está ligado a la entrega de dividendos cuando las empresas siguen políticas de "una acción, un voto". Cuando los votos están atados al pago de dividendos, los accionistas internos no pueden tener un 
control substancial de la empresa sin tener una propiedad substancial de los flujos de caja, lo que modera el desvío de los flujos de efectivo en relación con el pago de dividendos.

El segundo aspecto es el derecho de los acreedores. Esta dimensión es más compleja de analizar que la anterior, porque existen diferentes tipos de acreedores con diferentes tipos de interés, por tanto, los derechos de algunos acreedores pueden perjudicar los derechos de otros.

Otra razón para su complejidad es la existencia de dos estrategias de los acreedores para enfrentar el no pago de sus obligaciones (default), que son la liquidación o la reorganización de la empresa, las que necesitan diferentes leyes de protección. Según Hart (1995), el derecho básico de un acreedor con garantía es a tomar posesión de dicha garantía, ya sea para liquidarla o mantenerla, cuando los préstamos caen en default. En algunos casos, las leyes hacen difícil la reposición de la garantía por parte de los acreedores, porque esto podría llevar a las empresas a liquidarse, lo que es considerado como no deseado para la sociedad.

\section{Buen gobierno corporativo de la empresa}

\section{A. Principales códigos de gobierno corporativo}

Los códigos de buenas prácticas han surgido por la preocupación de articular procedimientos que garanticen los derechos de los accionistas y alienten un comportamiento responsable de los directorios (López-Iturriaga y Pereira, 2006).

Estos códigos abarcan aspectos tales como la divulgación de la información de la empresa, los deberes fiduciarios de los administradores o la misión de los órganos de gobierno.

El primer código de gobierno puede datarse de la década de los 70 en Estados Unidos, sin embargo, en el ámbito internacional, recién una década más tarde comienza a extenderse el movimiento 
de reforma del gobierno corporativo. En 1989, la Bolsa de Valores de Hong Kong publicó su primer Code of Best Practice, Listing Rules, y en 1991 la Asociación Irlandesa de Inversores redactó el Statement of Best Practice on the Role and Responsability of Directors of Publicly Listed Companies. En 1992, el Reino Unido publica el más destacado código, el Cadbury Report, por su influencia en la emisión de sugerencias de buen gobierno corporativo en otros países.

Por otro lado, en función del tipo de emisor, Aguilera y Cuervo-Cazurra (2009) clasifican los códigos en seis categorías: bolsas de valores (SEC, CNMV, TSX); Estado (gobierno de la nación o ministerios); asociaciones de consejeros (por ejemplo Japan Corporate Governance Committee); asociaciones de directivos (Institute of Directors en Sudáfrica); asociaciones de profesionales (Hong Kong Society of Accountants), y finalmente asociaciones de inversores (Investement and Financial Services Association Limited en Australia).

Para el caso de Estados Unidos, los recientes escándalos de Enron, Worldcom, Tyco, Adelphia, Global Crossing y la caída de Arthur Andersen, una de las cinco firmas más grandes de auditoría, sirvieron como catalizador para cambios legislativos, mediante Sarbanes-Oxley Act en 2002 y cambios regulatorios, como la nueva guía de gobiernos de NYSE y Nasdaq.

El contenido de las normas y códigos pueden ser clasificados dentro de una variedad de categorías, que van desde normas relacionadas con la certificación del CEO y CFO hasta normas que son un medio de control adecuado de la información que se revela y que es requerida por el mercado. La calidad de la información financiera presentada depende, en gran medida, de las normas de presentación de informes. En general estas normas están contenidas en los principios contables generalmente aceptados del país de procedencia de la empresa emisora.

En los últimos años, sin embargo, se ha buscado una convergencia de las normas de presentación de los estados 
financieros que traspasa las fronteras, como es el caso de Normas Internacionales de Información Financiera (NIIF) y las Normas Internacionales de Contabilidad Europea (NICE).

Otro tema relevante es la transparencia de las transacciones con empresas vinculadas. Angulo (2002) afirma que los accionistas y las partes relacionadas a las empresas desean conocer información que les ayude a determinar si el comportamiento de los directivos es acorde a todos los intereses de los accionistas y otros interesados y no una maximización de utilidad indebida hacia otras empresas vinculadas.

Desde una perspectiva cualitativa, tanto la legislación como las normas de buen gobierno corporativo han puesto énfasis en las características de las estructuras internas de la empresa. Tradicionalmente los auditores, abogados y analistas, permanecían fuera de la estructura del gobierno corporativo y evaluaban, desde su perspectiva de expertise, la condición financiera, la conducta legal y los prospectos de negocios de las empresas. Además cada uno de ellos tenía una relación diferente e independiente con la firma, por ejemplo, los auditores tenían el rol de verificar los reportes financieros según los principios contables generalmente aceptados. Por otro lado, los abogados estaban más identificados con la empresa y ayudaban a mantener su comportamiento dentro de los límites legales. Y finalmente los analistas no tenían relación con la empresa, solo ayudaban a sus clientes a realizar sus decisiones de inversión.

La ley SOX permite que estos tres actores importantes sean parte del gobierno corporativo de la empresa, es decir, que las juntas directivas tengan mayores directores independientes, que el comité de auditoría de la junta sea compuesto solamente por consejeros independientes con al menos un miembro con experiencia en contabilidad o finanzas. 
B. Sarbanes Oxley Act 2002 y el valor de la firma

Luego de una seguidilla de escándalos corporativos a fines del siglo XX, el Congreso de Estados Unidos aprobó la ley Sarbanes Oxley (SOX) en 2002.

Algunos de los cambios impuestos por SOX aumentan el rol de monitoreo de los directores independientes (tema expuesto con mayor detalle más adelante). La lista incluye: mayor independencia de los directores de la junta; definir estrictamente qué es independencia; existencia de comités de auditoría; nombramiento y compensación $\mathrm{y}$, por último, sesiones de directorio sin accionistas internos.

La motivación para esta serie de regularidad proviene de la creencia que las juntas directivas dominadas por directores independientes son buenas para todas las firmas, lo que tiene raíz en que antes de la regulación muchas firmas tuvieron directorios ineficientes.

Algunos estudios como Mulherin (2005) y Raheja (2005) demostraron que la composición de los directorios es endógena a las características individuales de cada firma y a su ambiente operativo. La teoría de los determinantes endógenos contiene que hay variaciones sistemáticas en la composición del directorio y las firmas tienden a buscar una estructura óptima que mantenga el equilibrio entre costos y beneficios del monitoreo externo.

Entonces, mientras la regulación puede ser beneficiosa para firmas que operan en entornos donde se necesita un alto monitoreo externo, también puede ser perjudicial para empresas con altos costos de monitoreo y que tienen poco beneficio del monitoreo de director externo. Wintoki (2007) tomó una muestra de alrededor de 1.500 empresas que transan en bolsa, de la que concluyó que las firmas con mayores costos de monitoreo externo se han visto poco beneficiadas por SOX.

Otros estudios han tratado de concluir acerca del beneficio (o costo) de SOX en el mercado como un todo. Siendo esto muy difícil, 
se ha hecho un alcance por medio de los modelos de valoración de activos de rendimientos esperados. Estos resultados han sido mixtos, por un lado Jain y Rezaee (2005) y Li et al. (2008) encontraron efectos positivos para la regulación en el mercado $\mathrm{y}$, por otro, concluyen que el efecto es negativo.

Zingales (2006) en su estudio de la competitividad de los mercados de capital en Estados Unidos encontró una caída dramática en IPO domésticas y extranjeras en los mercados accionarios entre 2000 y 2005.

Sin ir más lejos, podemos ver cómo estas nuevas regularidades han tenido impacto en los gobiernos corporativos. El objetivo es crear un entorno, donde habrá poco beneficio en la gestión de los ingresos y poco tipo de embustes financieros que podrían ayudar a inflar precios de las acciones en el corto plazo.

\section{El directorio}

A. Rol del directorio en el gobierno corporativo de la empresa

En general el directorio actúa como agente de los accionistas a cargo de dirigir la compañía. En grandes compañías, el directorio no está a cargo de manejar el día a día de la empresa, sino que maneja decisiones de alta relevancia y delega todas las otras responsabilidades a administradores.

Las principales responsabilidades de la junta directiva son:

- Contratar, evaluar y a lo mejor, despedir a los gerentes desde el CEO

- Votar en proposiciones de gran envergadura

- Votar en decisiones mayores de financiación

- Ofrecer consejos a los administradores

- Asegurar que las actividades financieras de la firma han sido reportadas a los accionistas, de forma correcta. 
El directorio puede ser considerado como el monitor interno más importante de la empresa, ya que representa la cúspide de la pirámide de jerarquía.

Hoy la prensa acusa a los directorios de insuficientes para vigilar el dinero de otros y que son muy burocráticos en la administración. Entonces ¿por qué existen los directorios?

Hermalin y Weisbach (1991) responden a base de un primer alcance, que son simplemente causa de la regulación. Entre la incorporación de leyes estatales y los requerimientos de los gobiernos de mercados accionarios, la mayoría de las firmas están obligadas a mantener un directorio que cumpla múltiples requerimientos, como debe tener un número determinado de miembros, debe cumplir con ciertas regularidades, puede necesitar varios comités y algunos directores pueden estar obligados a mantener cierta independencia con los administradores.

Sin embargo, no encuentran posible que los directorios sean solo eso y agregan, que si los gobiernos corporativos solo existieran por regularidades, entonces serían de gran costo para la empresa y, por tanto, en algún lugar del mundo dejarían de existir. Siendo esta la hipótesis, adicionan que por el alto costo que representan las empresas que mantengan directorios, lo harían en tamaños pequeños. Pero en la práctica, ocurre todo lo contrario, son grandes, incluso aún mayores al mínimo que las leyes exigen.

Dado esto, los autores dan una explicación más posible, y es que las juntas directivas son la solución a un problema de diseño organizacional del mercado, una institución endógena que aminora los problemas de agencias a los que se enfrenta una empresa. Desde esta perspectiva, afirman que es más útil estudiar cómo están estructurados y cuáles son sus funciones.

Una idea complementaria a la explicación recién expuesta radica en el problema mismo de la teoría de agencia, que fue expuesta en capítulos previos, pero brevemente se trata de los conflictos de interés entre accionistas y administradores, y cómo los primeros son capaces de monitorear las acciones de los segundos. Es 
así como nace la hipótesis para el nacimiento de las juntas, el monitoreo entre directores no era eficaz para que los accionistas confiaran en su administración, por tanto debía existir un ente intermedio.

Un tema importante del rol del directorio es su influencia en el desempeño de la firma. Para enfrentar este tema, separaremos el estudio en dos características principales de los directorios, su composición y su tamaño.

\section{B. El rol del director independiente}

Mittal (2011) afirma el surgimiento de los directores independientes del movimiento mundial de gobiernos corporativos. Estos han aumentado su presencia en las juntas directivas bajo el fundamento que pueden evitar fraudes y mala administración, el uso ineficiente de los recursos, la desigualdad y la falta de responsabilidad en la toma de decisiones y, además, como un presagio para lograr el balance entre intereses individuales, económicos y sociales.

El objetivo fundamental de los directores independientes es la imparcialidad. Las compañías desean identificar directores que sean capaces de realizar sus tareas sin ningún conflicto de interés en su tomas de decisiones. Para asegurar esto, hay ciertas pautas a tener en consideración al nombrar a directores independientes. La empresa debe adoptar una postura flexible en razón de las circunstancias vividas para satisfacer los criterios elegidos.

Según la Corporación Financiera Internacional (CFI), los directores independientes deben cumplir ciertos requerimientos. La norma que se pretende establecer asegura la integridad de la toma de decisiones, es decir, intereses contrarios a los de la empresa no deben interferir en las decisiones.

La definición de CFI indica que solo algunos son candidatos para ser directores independientes en una junta directiva. Ellos deben no haber sido empleados de la empresa o parte relacionada, 
considerando a los proveedores, consejeros o consumidores, en los cinco años previos a su candidatura.

Idealmente los directores independientes no deben haber sido directores de alguna otra compañía que cualquiera de sus directores sea miembro de la junta de esta compañía a la que postula. Así como tampoco ser familiar de algún director que esté en el directorio en los últimos años. Siendo aún más riguroso, el director independiente tampoco debe tener relación, familiar o laboral, con el auditor de la compañía.

Lawrence y Stapledon (1999) hacen una distinción de roles de monitoreo entre los directores ejecutivos y los no ejecutivos en la creación de valor de la empresa. Por otra parte, el Cadbury Report (1992) plantea que los directores no ejecutivos hacen dos contribuciones a los directorios. El primero es una revisión del desempeño de la junta y los ejecutivos $\mathrm{y}$, el segundo, es tomar posición de liderazgo en las decisiones que involucren conflictos de intereses.

Algunos investigadores de Estados Unidos han buscado evidencia de una supuesta conexión entre los directores independientes y el desempeño de la empresa. Estudios como el de Baysinger y Butler (1985) concluyó que la proporción de directores independientes tenía una correlación positiva con el retorno de capital. Sin embargo, Klein (1998), Bhagat y Black (1997, 1999) y Hermalin y Weisbach (1991) encontraron que una cantidad grande de directores independientes no podía predecir el desempeño de la empresa.

Por otro lado, un estudio de Agrawal y Knoeber (1996) concluyó que a mayor proporción de directores independientes, más lento era el crecimiento de la empresa.

Daily y Dalton (1992) hicieron un estudio a 100 empresas pequeñas de Estados Unidos y encontraron que los resultados financieros eran mejores en empresas con un mayor número de directores independientes. 
Finalmente, los estudios no han sido concluyentes en si los directores independientes añaden valor a la empresa. Estos estudios han buscado evidencia directa e indirectamente respecto de la relación de la composición de la firma y su desempeño financiero.

En el artículo de Karmel (2013) encontramos una mirada más reciente al modelo de director independiente y si cumple su rol de imparcialidad. Bajo el supuesto que los incentivos de los directores independientes estarían más alineados con los de los accionistas, ellos mantendrían mayor monitoreo en la junta directiva y velarían por la maximización de valor de los accionistas.

Sin embargo, las compañías han seguido fallando a pesar que la mayoría de las juntas directivas están compuestas por directores independientes y mantienen estructuras de comités relacionadas.

Ciertos estudios han demostrado que los directores independientes afectan negativamente a las empresas, porque son más propensos a aceptar prerrogativas de los administradores que los intereses de los accionistas. El estudio de Erkens (2009) examinó a 296 firmas en 30 países en la crisis financiera de 2008 y los resultados mostraron que las firmas con mayor número de directores independientes experimentaron peores retornos de acciones en el periodo de la crisis.

Los directores independientes por definición son "externos", por tanto, dependen de la información entregada por los administradores, lo que le da al CEO mayor poder que en el caso que el directorio tenga miembros "internos".

Karmel (2013) concluye su artículo afirmando que las cualidades que hacen a un director realmente independiente son la inteligencia, la experiencia y un fuerte sentido de la responsabilidad ética. La búsqueda de estas personas debe ser el objetivo de la selección del directorio. 


\section{Interlocking directorates}

Interlocking directorates o múltiple directorios es un tema estudiado desde 1932, cuando The modern corporation and private property fue publicada. Interlocking directorates se define como la relación entre corporaciones, creada por individuos que se sientan en más de una silla de juntas corporativas ${ }^{2}$. Esta acción es perfectamente legal, excepto en firmas que son competencia directa, sin embargo, no es deseable, ya que puede haber intercambio de información no pública y obtener beneficios sobrenormales.

Las primeras evidencias literarias fueron expuestas por Dooley (1969), concluyendo que 250 grandes empresas de Estados Unidos, en 1965, tendían a tener mayor relación entre directorios y que las empresas poco rentables también caían en esta práctica.

Mizruchi (1996) afirma que la relación de las juntas directivas se da tanto por los insiders, individuos afiliados a la empresa, y por outsiders, individuos que pertenecen a otra organización, como acreedores y accionistas minoritarios. Por definición, los outsiders crean necesariamente relación entre dos o más directorios.

Las posibles consecuencias del interlocking directorates que encontró Mizruchi (1996) son:

\section{a. COLUSIÓN}

Es legítimo preguntarse si el interlocking, específicamente entre competidores, facilita la colusión. En 1914, la asociación con más de un directorio se prohibió bajo el amparo de la ley "Clayton Act", sin embargo, en la década de 1960 ocurrió uno de los mayores escándalos de fijación de precio en la industria eléctrica.

\footnotetext{
${ }^{2}$ http://www2.ucsc.edu/whorulesamerica/power/corporate_community.html
} 
Adicionalmente, Baker y Faulkner (1993) afirman que esta ley tampoco evitó otras conspiraciones de fijación de precios ocurridas los años siguientes.

Con esta evidencia, entonces, nos preguntamos si el interlocking está motivado por los intentos de colusión, si son eficaces para facilitar la colusión o si en última instancia son irrelevantes.

En la literatura podemos encontrar a diversos autores que han examinado correlaciones y consecuencias de interlocks horizontales (dentro de una industria). Entre ellos podemos mencionar a Pennings (1980) y Burt (1983), que examinaron en firmas americanas la relación entre la concentración de la industria y los vínculos horizontales. Pennings encontró una relación positiva entre ambos y Burt encontró una función con forma de $\mathrm{U}$, donde el interlocking es mayor en industrias con niveles medios de concentración. Esto es consistente con la idea que, hasta cierto punto, la concentración facilita los lazos intraindustrias, pero las industrias altamente concentradas, debido a su reducido número de productores, no tienen incentivos para hacer interlock para fijar precios.

En relación con el desempeño y su mejora debido a los lazos de los directorios, Pennings no encontró asociación entre firmas relacionadas, con competidores y rentabilidad. Sin embargo, Carrington (1981), en firmas canadienses, encontró una relación positiva entre las tres variables.

\section{b. COOPTACIÓN Y MONITOREO}

Selznick (1949) define cooptación como la absorción de potenciales elementos disruptivos en la toma de decisiones de la empresa. Un ejemplo hipotético de cooptación, basado en Selznick, presentado por Thompson y McEwan (1959), es la invitación al directorio a un 
representante bancario, al que la firma le debe dinero. Entonces, ¿la cooptación sirve para aumentar la rentabilidad?

Los estudios al respecto han resultado ser mixtos. Pennings (1980), Carrington (1981) y Burt (1983) encontraron asociaciones generalmente positivas. Fligstein y Brantley (1992) encontraron una relación negativa entre interlocks y rentabilidad en una muestra de grandes empresas en USA. Así también, mencionamos otro caso, el de Bunting (1976), donde la evidencia relaciona interlock y desempeño en una función curvilínea, es decir, la rentabilidad aumenta con el aumento de los interlocks, pero hasta cierto punto, ya que luego la rentabilidad empieza a disminuir.

Otra interpretación para los hallazgos a la existencia de interlocks es un intento de monitoreo. Accionistas, banqueros y clientes frecuentemente esperan lograr representación en el directorio. Este fenómeno ha llevado a que la teoría sugiriera que los interlocks son instrumentos de control corporativo.

Berkowitz et al. (1979), Mizruchi (1982), Burt (1983) y Caswell (1984) identificaron lazos entre propiedad y representación directiva y han identificado el ingreso de banqueros a las juntas directivas en períodos posteriores a un bajo desempeño por parte de la firma, lo que es consistente con una perspectiva de monitoreo.

\section{c. LEGITIMIDAD}

Las juntas directivas son importantes para la reputación de la empresa. Cuando los inversionistas deciden invertir, consideran las fortalezas de la organización y la calidad de los administradores, entonces si se incorporan individuos que pertenecen a otras firmas exitosas al directorio, la firma señala al mercado que es legítima y es una buena inversión.

DiMaggio y Powell (1983) plantean la posibilidad que la firma alcanzará mayor financiamiento si es dirigida por individuos respetables. 
A pesar que el término de legitimidad ha jugado un rol importante en la teoría organizacional, el modelo, en lo que concierne a interlocking, ha sido poco estudiado $\mathrm{y}$, por tanto, difícil de comprobar. Finalmente, su predicción está relacionada con los recursos utilizados en su confección.

La mayoría de los análisis de los determinantes de los interlocks han implicado varias consecuencias. Como mecanismo de colusión, la existencia de interlocks facilita la comunicación, lo que puede significar traspaso de información entre competidores. Como mecanismo de cooptación, se asume que es para pacificar la gestión de los proveedores de recursos (ejemplo: banqueros). Y como mecanismo de monitoreo, proporciona a la firma influencia potencial en sus operaciones.

En un estudio más reciente, Chu y Davis (2011) estudiaron las propiedades del interlocking de grandes empresas de Estados Unidos entre 1999 y 2009. El análisis parte con un diagnóstico de la realidad de las empresas en el siglo XX, la que consta de tres partes.

Primero, al empezar el siglo XX, los bancos eran el nodo central de la conectividad directoral. Los fundamentos principales son que los bancos ponían a sus directores en las juntas directivas de otras empresas y cuando las corporaciones se hicieron más grandes y comenzaron a depender en gran medida de sus ingresos financieros, los bancos iniciaron el reclutamiento de CEO bien conectados para servir como consejeros externos en sus propias juntas.

Segundo, existía un "círculo interno" identificable de directores que eran particularmente importantes en el dinamismo de la red. Según Mike Useem (1984), estos “diplomáticos corporativos" tenían una perspectiva distintivamente cosmopolita, que abarcaba los amplios intereses de las empresas y no solo los intereses de un sector en particular, y eran más propensos que otros directores a participar en las organizaciones políticas y de servir en el servicio público (antes o después de sus carreras de director). 
Finalmente, la tercera conclusión es que los interlocks tienen un misterioso pequeño mundo, en el que todo el mundo parece conocerse o tener amigos en común. Mills (1956) afirma que como una elite no organizada, todos los miembros parecen conocerse unos con otros, siendo perfectamente normal trabajar juntos y compartir muchas organizaciones. No son conspiraciones, pero sus decisiones no son sabidas públicamente y sus operaciones son más manipulativas que explícitas.

En una revisión para los 80 la posición central de los bancos comenzó a declinar y ya para fines de 1990, los bancos ya no dominaban el ranking de las empresas más centrales. Davis y Mizruchi (1999) atribuyen como causa principal que las empresas se voltearon al mercado de capitales para su financiamiento, por tanto, los bancos comerciales empezaron a mirar otros mercados, disminuyeron su número de miembros en las juntas directivas y dejaron de reclutar a ejecutivos bien conectados. Por otra parte, para Neuman (2008), la consolidación del sector bancario mediante líneas geográficas e industriales disminuyó la cantidad de bancos con los cuales asociarse.

El círculo interno también había cambiado. Mills (1956) escribió que la elite eran hombres blancos y de buena crianza. Sin embargo, las presiones sociales para tener una representatividad más diversa permitieron la promoción de un grupo "demográficamente atractivo" al directorio. Para 1999, muchos diplomáticos directivos eran mujeres y cuatro de los mejores directores eran afroamericanos.

Siguiendo el estudio de Chu y Davis (2011), el pequeño mundo de los directores, que parecía ser una ley que operaba independiente de las otras características, dejó de ser tan pequeño. Davis et al. (2003) reportó que la geodésica media entre las mayores 600 o más corporaciones estadounidenses era 3,38 en 1982, 3,46 en 1990 y 3,46 en 1999, mientras que la línea geodésica entre los directores fue 4,27, 4,30, y 4,33, respectivamente. 
A partir del siglo XXI han surgido potenciales retos a la estructura del interlocking de los gobiernos corporativos en Estados Unidos.

Desde una perspectiva estructural de las juntas de gobierno, tres eventos han reducido el atractivo de pertenecer a uno. El primer caso es la caída de Nasdaq en 2000, el que fue seguido por escándalos de conflictos de interés en el sistema corporativo de Estados Unidos. En consecuencia, el Congreso aprobó SarbanesOxley Act en 2002, lo que ha contribuido a incrementar la responsabilidad de los directores, especialmente los que pertenecen al comité de contabilidad. Por último, la gran depresión de 2007 y el declive de los mercados, incidieron en aumentar la presión de los directores.

Este siglo también puso en evidencia el avance de la consolidación bancaria, cuando los cuatro mayores sobrevivientes (JP Morgan Chase, Bank of America, Citigroup y Wells Fargo) adquirieron a la mayoría de sus rivales. La crisis financiera de 2008 puso más presión en la consolidación, ya que los bancos en mayores problemas fueron presionados para fusionarse con algunos rivales de la industria bancaria.

Por último, ha existido una disminución de los sistemas corporativos en los últimos años. En 2009, Estados Unidos tenía la mitad del número de corporaciones públicas que tenía en 1997, debido a que la oferta pública inicial no pudo ir al ritmo de adquisiciones y exclusiones. El famoso "crepúsculo de las corporaciones públicas" y el surgimiento de nuevas formas de financiamiento, como el capital privado, evidencia que los interlocks han sido una característica específica de los gobiernos corporativos en el siglo XX, no como un elemento permanente del capitalismo. 
D. Mujeres en el directorio y la toma de decisiones

La diversidad de género es un tema estudiado a nivel mundial. En Estados Unidos en 2007, los directorios con mujeres como miembros eran alrededor del $14,87 \%$ de las empresas en Fortune 500. En Australia, Canadá, Japón y Europa se estima que el 8,7\%, $10,6 \%, 0,4 \%$ y $8 \%$, respectivamente, corresponde a empresas con mujeres en el directorio (EOWA 2006 ${ }^{3}$ ).

Adams y Ferreira (2009) afirman que esta situación está por cambiar, ya que los gobiernos corporativos están bajo presión para elegir directores mujeres. Muchas propuestas de Estados explicitan la importancia de la diversidad de género en los directorios.

Por ejemplo en Suecia, si las empresas no tenían voluntariamente una reserva del $25 \%$ de su composición directiva destinada a mujeres, eran amenazados de hacer la diversidad de género un tema legal (Medland, 2004). En Noruega, el caso más extremo, desde 2008 todas las empresas que transan en bolsa deben tener una cuota de $40 \%$ de directivos mujeres o disolverse.

La mayoría de estas iniciativas están basadas en que la presencia de mujeres en los directorios puede afectar la toma de decisiones en diferentes formas. Fitzsimmons (2012) afirma que los reportes implican la paridad de género en las juntas directivas, como si esto naturalmente trajera beneficios. En contraste, académicos han encontrado que la diversidad de género puede significar un peor resultado en el desempeño de la firma, aun si hay evidencia de una relación positiva entre la diversidad de género y el desempeño de la empresa, no es claro si la diversidad mejora el desempeño o viceversa.

Adams y Ferreira (2009) realizan un estudio en el que pretenden explicar tres preguntas. La primera, ¿ciertas medidas del directorio, como asistencia y asignación de comité, varían con la

\footnotetext{
Leadership.

${ }^{3}$ http://www.catalyst.org/knowledge/2006-eowa-australian-census-women-
} 
diversidad de género? En segundo lugar, ¿la composición de la directiva afecta medidas de gobernabilidad, como las adquisiciones y compensación de los CEO? Y finalmente, ¿la diversidad de género importa tanto para afectar el desempeño de la firma?

El estudio concluye respecto de la primera pregunta que las mujeres se comportan, aparentemente, de forma diferente a los hombres. Específicamente, parecen tener menos problemas de asistencia. La otra característica a medir fue la asignación a comités, donde se encontró que las mujeres, probablemente, participen más en comités relacionados con monitoreo que los hombres. De hecho, las mujeres es más probable que sean asignadas a comités de auditoría y de gobiernos corporativos, y es menos probable que pertenezcan a un comité de compensación.

Respecto de las adquisiciones y compensación de los CEO, se encontró que los directorios más diversos son más factibles de mantener a sus CEO a un peor desempeño del precio de la acción. El movimiento de CEO es más sensible al desempeño del precio de la acción en empresas que tienen mayor número de mujeres en sus directorios. El estudio también concluye que los directores que pertenecen a juntas más diversas reciben una compensación más equitativa. Sin embargo, no se encontró una relación estadística entre diversidad de género y nivel de compensación.

La evidencia de la relación entre la diversidad de género y el desempeño de la firma ha sido más difícil de demostrar. La correlación entre diversidad de género con el valor de la firma o con el desempeño operativo, parece ser positivo en unas primeras luces, sin embargo, desaparece al incluir ciertas variables y problemas de causalidad. Las firmas tienen un peor desempeño a medida que aumenta la diversidad de género. Este resultado es consistente con Almazán y Suárez (2003), que plantean que a un mayor monitoreo puede disminuir el valor de los accionistas.

Por otro lado, Fitzsimmons (2012) estudia por qué no es suficiente para las empresas "solo sentar" en sus directorios a 
mujeres. En vez de eso, deberían fomentar ambientes propicios antes de obtener algún beneficio de la diversidad de género.

Algunos estudios se preguntan qué detiene a las juntas directivas de tener más diversidad de género y cuándo las empresas son exitosas, después de una diversificación de género. Estas dos preguntas están relacionadas con las metas de la diversidad de género puestas por el directorio. Es decir, que las condiciones que guían el éxito de la diversificación de las juntas no son necesariamente las mismas que guían a una mejora en la gobernabilidad.

Un estudio reciente de Ernst and Young ${ }^{4}$ (2012) revisa un progreso de la diversidad de género de las juntas directivas en Estados Unidos. El que concluyó que las mujeres están entrando a los directorios, como un porcentaje de nuevos miembros, a una tasa que ha crecido rápidamente en los últimos años y que las juntas que ya mantienen una mujer en su directorio es más probable que permita el ingreso de otra.

La tabla 1 representa la tasa de crecimiento de las mujeres como nuevos miembros. Más de 1.800 puestos de directores, actualmente llenado por mujeres, aproximadamente el $40 \%$ se unió en los últimos cinco años.

Las mujeres, a pesar que los estudios no encuentran evidencia significativa entre diversidad de género y desempeño del directorio, encuentran en la literatura razones de por qué es necesaria su membrecía.

Entre ellas destacan: aumentar la diversidad de opinión en la sala de junta directiva (Catalyst, $199^{5}$ ), mujeres dan mejores estrategias (Bilimoria, 2000), influyen la toma de decisiones y el estilo de liderazgo organizacional (Rosener 1990) y aseguran un mejor comportamiento en la sala de junta (Across the board, 1994).

\footnotetext{
${ }^{4}$ http://www.ey.com/Publication/vwLUAssets/Getting_on_board/\$ FILE/Getting_on_board.pdf

${ }^{5} \mathrm{http}: / /$ www.catalyst.org/knowledge/1995-catalyst-census-women-boarddirectors-fortune-500
} 
Finalmente, las mujeres han contribuido con la gobernabilidad a reducir el poder de los CEO y las directoras externas contribuyen con una mirada independiente al directorio y demuestran cómo la intervención de una mujer director puede cambiar la dirección estratégica de una empresa (Selby, 2000).

\section{Tabla 1}

Mujeres como porcentaje de nuevos miembros

(Ernst and Young, 2012)

\begin{tabular}{|c|c|}
\hline $\begin{array}{c}\text { AÑO DE UNIRSE A LA JUNTA } \\
\text { DIRECTIVA }\end{array}$ & $\begin{array}{c}\text { MUJERES COMO PORCENTAJE DE NUEVOS } \\
\text { MIEMBROS DE LA JUNTA DIRECTIVA }\end{array}$ \\
\hline $2000-2009$ & 14 \\
\hline $1990-1999$ & 11 \\
\hline $1980-1989$ & 6 \\
\hline $1970-1979$ & 2 \\
\hline $1960-1969$ & 3 \\
\hline 2010 & 16 \\
\hline 2011 & 18 \\
\hline 2012 & 21 \\
\hline
\end{tabular}

\section{Convergencia de gobiernos corporativos internacionales}

Existen diferencias fundamentales en los sistemas de gobiernos corporativos en los países del mundo. Los primeros países estudiados, aparte de Estados Unidos, fueron Alemania, Japón y Reino Unido.

En los años 90, Estados Unidos tenía un sistema notoriamente basado en el mercado. En cambio, Japón se basaba en las relaciones. Los defensores de esta idea argumentaban que era un sistema superior de control externo y que así los ejecutivos no caían en presiones de corto plazo, ocasionadas por el mercado. 
En el ámbito legal, Estados Unidos y Reino Unido mantienen una severa protección legal a sus inversionistas. En cambio, Japón y Alemania están caracterizados por un sistema débil, pero con una alta concentración en la participación del capital.

Shleifer y Vishney (1997) y Hansmann y Kraakman (2000) plantean que los sistemas de Estados Unidos, Japón y Alemania muestran una significativa convergencia de ideas en los mecanismos de gobierno corporativo.

Wojcik (2001) estudió la empresas alemanas entre 1997 y 2000 , encontró que la concentración de propiedad había disminuido significativamente durante el periodo, que las participaciones cruzadas empezaron a disolverse y que las instituciones del sector financiero disminuyeron la cantidad de accionistas mayoritarios en sus directorios. En conclusión, el sistema alemán se estaría moviendo a un sistema anglosajón.

Los países se están moviendo a prácticas de código de buen gobierno corporativo, guiados por el sistema anglosajón. Dahya et al. (2002) reportan evidencia de grandes cambios en la estructura del directorio en Reino Unido, la que es favorable a la adopción de dichos códigos. Sin embargo, existe evidencia por parte de los gobiernos italianos que han intentado aumentar la protección a los inversionistas minoritarios por medio del fomento de un mayor activismo de los inversionistas institucionales, pero no ha sido exitoso.

Liu (2001) expone que las leyes de valores en China y Taiwán están verdaderamente influenciadas por la ley común. En China, el objetivo es disminuir la liquidación de activos por los directores y gerentes de las empresas de propiedad estatal. En Taiwán, es disminuir la expropiación a accionistas minoritarios por los fundadores de empresas cotizantes.

Aún no es clara la convergencia global, sin embargo, el tiempo dirá cuál será el resultado final de la convergencia del sistema de gobierno corporativo. 


\section{Conclusión}

Existe una extensa literatura que estudia a los gobiernos corporativos, tanto en Estados Unidos como en el resto del mundo. Las opiniones de los investigadores también son diversas, al igual que algunos resultados. Estas diferencias son producidas por la evidencia empírica, donde se recogen los datos para la realización de los estudios.

Uno de los componentes más importantes para la existencia de estas diferencias radica en los sistemas legales de cada país y el entorno legal donde opera la firma. Los autores plantean que entender los sistemas legales es primordial para conocer el funcionamiento de las finanzas corporativas y los gobiernos corporativos en los diferentes países del mundo.

Para el caso de Estados Unidos, el origen anglosajón de su sistema legal ha permitido que los gobiernos corporativos mantengan una alta protección a sus inversionistas, ya sean los acreedores o accionistas, en comparación con países con origen legal en la ley civil. Empíricamente se ha demostrado que una vigorosa protección a los inversionistas está asociado con un gobierno corporativo eficiente.

Un importante mecanismo interno, que ha sido tema en los últimos años por las fallas en los gobiernos corporativos, es la junta directiva o el directorio. Tres temas que en la práctica se han desarrollado son: los directores independientes, interlocking directorates y la diversidad de género.

Los directores independientes han nacido con el objetivo de ser imparciales a la hora de tomar decisiones. Como tareas se destacan evitar fraudes y mala administración, el uso indebido de recursos, las desigualdades y la falta de responsabilidad en la toma de decisiones. Algunas cualidades que se buscan en ellos son la inteligencia, la experiencia y la responsabilidad ética.

Los interlocks ocurren cuando un director pertenece a más de una junta directiva. Algunas consecuencias son la colusión, que 
facilita la comunicación entre empresas, la cooptación y monitoreo, que pacifica la gestión de los proveedores de los recursos e influye en las operaciones, y la legitimidad, que da a las empresas reputación al momento de buscar acreedores y formas de financiamiento.

La diversidad de género en los directorios ha sido aceptada y el número de mujeres como miembros en juntas directivas ha ido aumentando con el tiempo. Empíricamente, las mujeres tienen mejor asistencia a las reuniones y participan en comités de monitoreo más que los hombres. Los CEO que forman parte de empresas con mujeres como directores, reciben compensaciones más equitativas. Sin embargo, la evidencia empírica no ha sido clara en qué pasa con el desempeño de las firmas y la diversidad de género, pero en la literatura se encuentran razones para apoyar su membrecía.

Finalmente, se ha demostrado que los países en el mundo están ejerciendo códigos de buen gobierno corporativo basados en los orígenes de ley común, por tanto, nos estamos acercando a una convergencia global en cuanto a las prácticas de gobiernos corporativos. 


\section{Referencias}

Across the BoARD, (1994), “Gender Chill?”, September, 1.

ADAMS, R.B., and D. FERREIRA (2009), "Women in the boardroom and their impact on governance and performance", Journal of Financial Eeconomics, Vol. 94(2), pp. 291-309

Agrawal, A. and C.R. KNOEBer (1996), "Firm performance and mechanisms to control agency problems between managers and shareholders", Journal of Financial and Quantitative Analysis, Vol. 31(3), pp. 377-397.

Aguilera, R.V. and A, Cuervo-Carruza (2009), "Codeso of good governance", Corporate governance: an international review, Vol. 17(3) pp. 376-387.

AlmazAn, A. and J. SuÁREZ (2003), "Entrenchment and severance pay in optimal governance structures", The Journal of Finance, Vol. 58(2), pp. 519-547.

ANGULO, J.A.G. (2002), "Información contable, auditoría y gobernanza empresarial", Ekonomiaz: Revista Vasca de Economía, Vol. (50), pp.28-53.

BAKER, W.E., and R.R. FAULKNER (1993), "The social organization of conspiracy: Illegal networks in the heavy electrical equipment industry", American Ssociological Review, pp. 837-860.

BAYSingeR, B.D., and H.N. BUtLER, (1985), "Corporate governance and the board of directors: Performance effects of changes in board composition", Journal of Law, Economics, \& Organization, Vol. 1(1), pp. 101-124.

BEBCHUK, L.A. (1999), "A rent-protection theory of corporate ownership and control", National Bureau of Economic Research, (N . W 7203).

BECHT, M. (1999), "European corporate governance: Trading off liquidity against contro",. European Economic Review, Vol. 43(4-6), pp. 1071-1083.

BENNEDSEN, M. and D. Wolfenzon (2000), "The balance of power in closely held corporations", Journal of Financial Economics, Vol. 58(1-2), pp.113-139. 
Berger, P.G. and E. OfEK (1996), "Bustup takeovers of value destroying diversified firms", The Journal of Finance, Vol. 51(4), pp. 1175-1200.

Berle, A. and G.C. MEANS (1932), "The modern corporation and public property", NY: Harcourt, Brace \& World.

Berkowitz S.D, P. CARrington, Y. Kotowitz and L. WA-VERMAN (1979), "The determination of enter-prise groupings through combined ownership and directorship ties", Social Network, Vol. 1(4), pp. 391-413.

BHAGAT, S. and B. BLACK (1997), "Do independent directors matter?", Journal of Finance, Vol. 52 (3), pp. 1212-1212.

BhAGAT, S. and B. BLACK (1999), "The uncertain relationship between board composition and firm performance", The Business Lawyer, Vol. 54 (3), pp. 921-963.

BILIMORIA, D. (2000), "Building the Business Case for Women Corporate Directors. Chapter in Women on Corporate Boards of Directors: International Challenges and Opportunities". Eds. R. J. Burke and M. C. Mattis. Dordrecht, The Netherlands: Kluwer Academic Publishers, pp. 25-40.

BUNTING D. 1976, "Corporate interlocking. Part III. Interlocks and return on investment", Directors and Boards, Vol. 1(fall), pp. 4-11

BURT, R. (1983), "Corporate profits and cooptation: Networks of market constraints and directorate ties in the American economy", New York: Academic Press.

CAdBury Report. (1992), "Report of the Committee on the Financial Aspects of Corporate Governance: European Corporate Governance Institute”.

CARrington, P.J. (1981), "Horizontal co-optation through corporate networks". Unpublished PhD Dissertation, University of Toronto, Toronto.

CASWELl J.A. (1984), "An institutional perspective on corporate control and the network of inter-locking directorates", J. Econ. Iss, Vol. 18, pp. 619-626.

Catalys Census (1995), "Female board directors of the Fortune 500". 
Claessens, S. and B. Yurtoglu (2012), "Corporate governance and development: An update", A Global Corporate Governance Forum Publication, Focus 10.

COFFEE, J.C. (2006), "Gatekeepers: The professions and corporate governance", Oxford University Press on Demand.

ChU, J.S.G. and G.F. DAvis (2011), "Who Killed the Inner Circle?", The Breakdown of the American Corporate Elite Network, 1999-2009, Paper 1.

Chung, K.H., J.S. KIM, K. PARK and T. SunG (2012), “Corporate governance, legal system, and stock market liquidity: Evidence around the world", Asia-Pacific Journal of Financial Studies, Vol. 41(6), pp. 686-703.

DAHYA, J., J.J. MCCONNELl and N.G. Travlos (2002), "The Cadbury committee, corporate performance, and top management turnover", The Journal of Finance, Vol. 57(1), pp. 461-483.

DAILY, C.M. and D.R. DALTON (1992), “The relationship between governance structure and corporate performance in entrepreneurial firms", Journal of Business Venturing, Vol. 7(5), pp. 375-386.

DAVIS, G.F. and M..S MizRUCHI (1999), "The money center cannot hold: Commercial banks in the US system of corporate governance", Administrative Science Quarterly, Vol. 44(2), pp. 215-239.

Davis, G.F., Mina Yoo, and E, Baker Wayne (2003), "The small world of the American corporate elite, 1982-2001”, Strategic Organization, Vol. 1, pp. 301-326.

DENIS, D.K. and J.J. MCCONNELL (2003), "International corporate governance", Journal of Financial and Quantitative Analysis, Vol. 38(1), pp. 1-36.

DiMaggio P.J. and W.W. Powell (1983), "The iron cage revisited: institutional isomorphism and collective rationality in organizational fields", $\mathrm{Am}$. Sociol. Rev, Vol. 48, pp. 147-160.

Donaldson, G. (1994), "Corporate Restructuring Managing the Change Process from Within", Harvard Business School Press.

Dooley, P.C. (1969), "The interlocking directorate", The American Economic Review, Vol. 59(3), pp. 314-323. 
EAOWA (2006), “Australia census of women in leadership".

ERKens, D., M. Hung and P. MAtos (2009), "Corporate governance in the recent financial crisis: Evidence from financial institutions worldwide", SSRN Paper, 1397685.

FITZSIMMONS, S. (2012), "Women on boards of directors: Why skirts in seats aren't enough", Business Horizons, Vol. 55, pp. 557-566.

Fligsteain N. y P. BRAntley (1992), "Bank control, owner control, or organizational dynamics: Who controls the large modem corporation?", American Sociological Review, Vol. 98, pp. 280-307.

Grossman, S.J. and O.D. HART (1988), "One share-one vote and the market for corporate control", Journal of Financial Economics, Vol. 20, pp. 175-202.

Hansmann, H. and R. KRAAKMAN (2000), "The End of History for Corporate Law",Yale Law School Working 235; NYU Working 013; Harvard Law School Discussion Paper 280; Yale SOM Working ICF-00-09.

HART, O. (1995), "Corporate governance: some theory and implications", The Economic Journal, Vol. 105(430), pp. 678-689.

Hermalin, B.E. and M.S. Weisbach (1991), "The effects of board composition and direct incentives on firm performance", Financial Management, pp. 101-112.

Holderness, C.G. and D.P. SHEEHAN (1991), "Monitoring an owner: The case of Turner Broadcasting”, Journal of Financial Economics, Vol. 30(2), pp. 325-346.

JAIN, P.K. and Z. REZAEE (2006), "The Sarbanes-Oxley Act of 2002 and CapitalMarket Behavior: Early Evidencae", Contemporary Accounting Research, Vol. 23(3), pp. 629-654.

JENSEN, M.C. (1993), “The modern industrial revolution, exit, and the failure of internal control systems", The Journal of Finance, Vol. 48(3), pp. 831-880.

KAPLAN, S.N. and L. Zingales (1997), "Do investment-cash flow sensitivities provide useful measures of financing constraints?", The Quarterly Journal of Eeconomics, Vol. 112(1), pp. 169-215. 
KARMEL, R.S. (2013), "Is the independent director model broken?", Seattle UL Rev., Vol. 37, pp. 775.

KIRKPATRICK, G. (2009), "The corporate governance lessons from the financial crisis", OECD Journal: Financial Market Trends, Vol. 2009(1), pp. 61-87.

KLEIN, A. (1998), "Firm performance and board committee structure", The Journal of Law and Economics, Vol. 41(1), pp. 275-304.

La Porta, R., F. López de Silanes, A. Shleifer y R. Vishny, R. (1998), "Law and finance", Journal of Political Economy, Vol. 106(6), pp. 1113-1155.

La Porta, R., F. Lopez-De-Silanes and A. Shleifer (1999), "Corporate Ownership Around the World", Journal of Finance, Vol. 54(2), pp. 471-517.

LA Porta, R., F. Lopez-DE-Silanes, A. Shleifer y R. Vishny (2000), "Investor protection and corporate governance", Journal of Financial Economics, Vol. 58(1), pp. 3-27.

LAWRENCE, J., and G. STAPLEDON (1999), "Do independent directors add value?”, Melbourne: Centre for Corporate Law and Securities Regulation, University of Melbourne.

LI, H., M. PINCUS AND S.O. REGO (2008), "Market reaction to events surrounding the Sarbanes-Oxley Act of 2002 and earnings management", The Journal of Law and Economics, Vol. 51(1), pp. 111-134.

LIU, L.S. (2001), "Chinese Characteristics Compared: A Legal and Policy Perspective of Corporate Finance and Governance in Taiwan and China”, http://dx.doi.org/10.2139/ssrn.273174

LOPEZ-IturriagA, F.J., and M.L. Pereira DO CARMo (2006), "Análisis internacional de los códigos de buen gobierno", Universia Business Review, Vol. (11), pp. 10-21

Medland, D. (2004), "Small steps for womankind", Corporate Board Member Europe, Winter. 
Merton, R.C., and Z. Bodie (1995), "A conceptual framework for analyzing the financial system", The global financial system: A functional perspective, pp. 3-31.

MiLLS, C. (1956), "Wright: The power elite", New York.

MitTAL, P. (2011), "The Role of Independent Directors in Corporate Governance”, NUJS L. Rev., Vol. 4, pp. 285.

MizRUCHI M.S. 1982, “The American Corporate Network: 1904-1974”, Beverly Hills: Sage.

MizRUCHI, M.S. (1996), "What do interlocks do? An analysis, critique, and assessment of research on interlocking directorates", Annual Review of Sociology, Vol. 22(1), pp. 271-298.

Morck, R., A. SHLEIFER and R.W. VishnY (1989), "Alternative Mechanisms for Corporate Control", American Economic Review, Vol. 79(4), pp. 842-852.

MulHerin, J.H. (2005), "Corporations, collective action and corporate governance: One size does not fit all”, Public Choice, Vol. 124(1-2), pp. 179-204.

Neuman, E.J., G.F. DAVIS and M.S MizRUCHI (2008), "Industry consolidation and network evolution in US global banking, 1986-2004", in Network Strategy, (pp. 211-245), Emerald Group Publishing Limited.

OECD, O. (2004), "The OECD principles of corporate governance", Contaduría y Administración, Vol. (216), pp. 183-194,_http://dx.doi.org/10.22201/fca $24488410 \mathrm{e} .2005 .562$.

Pennings, J.M. (1980), “Interlocking Directorates”, San Francisco: Jossey Bass.

RAHEJA, C. G. (2005), "Determinants of board size and composition: A theory of corporate boards", Journal of Financial and Quantitative Analysis, Vol. 40(2), pp. 283-306.

RAJAN, R.G. (1998), "The past and future of commercial banking viewed through an incomplete contract lens", Journal of Money, Credit and Banking, Vol. 30(3), pp. 524-550. 
Rosener, J. B. (1990), “Ways Women Lead", Harvard Business Review, Vol. 68, pp. 119-125.

SELBY, C.C. (2000), "From Male Locker Room to Co-executive Director Boardroom: A Twenty-five Year Perspective", in R.J. Burke and M. C. Mattis (eds.), Women on Corporate Boards of Directors, Kluwer Academic Publishers, The Netherlands, pp. 97-109.

SELZNICK, P. (1949), "TVA and the Grass Roots: a Study in the Sociology of Formal Organization", Berkeley: University of California Press.

SHLEIFER, A., and R. ViSHNY (1997), "A survey of corporate governance", Journal of Finance, Vol. 52(2), pp. 737-782.

Smith, A. (1776), "1776 The Wealth of Nations". Oxford University Press, Oxford.

THOMPSON J.D. and W.J. MCEWAN (1959), "Organizational goals and environment: Goal setting as an interaction process", American Sociological Review, Vol. 23(1), pp. 23-31.

UsEem, E.L. (1984), "Education and high-technology industry: the case of Silicon Valley", Economics of Education Review, Vol. 3(3), pp. 215-221.

VEASEY, E.N. (1993), “The Emergence of Corporate Governance as a New Legal Discipline”, Business Lawyer, Vol. 48(4), pp. 1267-1270.

WinTOKI, M.B. (2007), "Corporate boards and Regulation: The effects of the Sarbanes-Oxley Act and the exchange listing requirements on firm value", Journal Corporate Finance, Vol. 13(2-3), pp. 229-250.

WoJcIK, D. (2001), "Change in the German Model of Corporate Governance: Evidence from Blockholdings 1997-2001".

ZINGALES, L. (1994), "The Value of the Voting Right: A Study of the Milan Stock Exchange Experience", Review of Financial Studies, Vol. 7(1), pp. 125-148.

ZIngales, L. (2006), “Is the U.S. Capital Market Losing its Competitive Edge?”, University of Chicago, NBER and CEPR. 
